\title{
Fabrication, Rheology and Antioxidant Activity of Palm Esters-based Emulsions Loaded with Tocotrienol
}

\author{
S. H. $\mathrm{NG}^{1 *}$, B. MAHIRAN ${ }^{2}$ AND I. ZAHARIAH ${ }^{3}$
}

\begin{abstract}
Palm oil esters are wax esters derived from palm oil and oleyl alcohol. Palm oil esters have many applications due to their luxurious moisturizing properties, non-greasy and blend easily with fragrances and colours when applied on skin surface. The aim of this research was to fabricate palm esters-based emulsions cream for topical delivery, characterise the rheological properties and in vitro antioxidant activity of the palm esters-based emulsions system.

Emulsions containing palm oil esters loaded with tocotrienol were obtained in two stages, with propagation of rotor-stator at 6000 r.p.m. for $5 \mathrm{~min}$ and further emulsified using an ultrasound at various acoustic amplitudes for another $5 \mathrm{~min}$. A stress/rate controlled Kinexus Rheometer with a temperature controller was used to measure the rheological properties of the emulsion. Rheology measurements were performed at $25.0^{\circ} \mathrm{C} \pm 0.1^{\circ} \mathrm{C}$ with $4 \% / 40 \mathrm{~mm}$ cone and plate geometry. The in vitro antioxidant activity was investigated using UV-Vis spectrophotometer.

The yield stress of the emulsions increased with increasing acoustic amplitudes. The viscoelasticity of the emulsions were enhanced by the increase in the oil and surfactant concentrations. The emulsions with higher oil phase concentration $[30 \%(\mathrm{w} / \mathrm{w})]$ showed greater elasticity which implied strong dynamic rigidity of the emulsions. The cohesive energy increased significantly with surfactant concentration especially for the emulsions with $30 \%(\mathrm{w} / \mathrm{w})$ oil phase concentration. The palm oil esters emulsions containing tocotrienol gave higher Trolox equivalent antioxidant capacity values which implied higher antioxidant capability.

The tocotrienol in emulsion with $30 \%(\mathrm{w} / \mathrm{w})$ dispersed phase showed that they were the most stable with longest shelf life and exhibited greater inhibitory effects on the ABTS ${ }^{++}$.
\end{abstract}

Key words: Emulsion; rheological properties; antioxidant activity; yield stress; cohesive energy; palm oil esters; tocotrienol

Palm oil is produced from the fruit of oil palm (Elaeis guineensis) which is grown in mass plantations in tropical countries such as Malaysia, Indonesia and Nigeria. The oil consists of $95 \%$ triglycerides and $5 \%$ diglycerides whereby carbons of the carboxyls range from 10-20 with or without double bonds (Tanaka et al. 2008). Palm oil esters (POEs) are a constituent of modified form of palm olein oil known simply as palm oil. Desirable characteristics of fat esters including non-toxicity, good fat solubility properties and excellent wetting at interfaces (Radzi et al. 2006) but without the greasy feeling when applied on the skin surface; these have attracted the attention of the industry. The emollient effect of POEs had been proven thereby making this oil highly recommendable for its incorporation into the topical preparation as oil phase. Thus, palm oil esters are excellent ingredient to be used in cosmeceutical and pharmaceutical formulations.

\footnotetext{
${ }^{1}$ School of Pharmacy, International Medical University, 57000 IMU Bukit Jalil, Kuala Lumpur, Malaysia

${ }^{2}$ Department of Chemistry, Faculty of Science, Universiti Putra Malaysia, 43400 UPM Serdang, Selangor, Malaysia

${ }^{3}$ Sime Darby Research Sdn. Bhd Carey Island, 42960 Pulau Carey, Selangor, Malaysia

*Corresponding author (e-mail: sookhan_ng@imu.edu.my)
} 
Nanoemulsions are emulsion with droplet size in the range $20 \mathrm{~nm}-200 \mathrm{~nm}$ (Solans et al. 2003). They are independent of molecular size of the hydrophilic solute and the nature of the aqueous phase. In addition, nanoemulsions delivery system was independent of animal skin characteristics such as the stratum corneum thickness and the follicle-type (Wu et al. 2001). Thus, nanoemulsions due to their extremely small size are suitable to be used as delivery system in cosmeceuticals. However, nanoemulsions are only kinetically stable and therefore, it is also a very fragile system by nature (Tadros et al. 2004). As they are transparent and usually very fluid, the slightest sign of destabilization easily appears. They become opaque and creaming may be visible. Thus, stability of the nanoemulsion is a critical factor to be analysed. The achievement of developing long time stability of cosmetic products (3-years shelf life) is often difficult and deeply affects costs in the development of new formulations.

Rheology is an independent scientific discipline: studying the deformability, and flow properties of a matter under an applied stress or strain is revealed by McClements (1999). Owing to the fact that rheology can give a better picture of the behaviour of a material, it is therefore widely used as a tool to test the texture and flow behaviour of industrial products especially in the processing industries such as food (Lorenzo et al. 2008), cosmetics (Bummer \& Godersky 1999), pharmaceuticals (Zumalacarregui et al. 2004), polymer (Karg et al. 1985), coating (Kikic et al. 1979), and oil processing (Martin et al. 2006). The rheological results also enable scientists to estimate the product's quality such as elasticity, viscosity, deformability, storage, shelf life including intermolecular interactions due to ultra-sensitivity at microstructure of materials.

Antioxidants neutralize damaging free radicals by quenching reactive molecules and, thus protecting cells from both endogeneous stress (byproducts of cellular energy) and exogenous stressors (ultraviolet light, pollution, cigarette smoke etc.) (Choi \& Berson 2006). Tocotrienol are fat-soluble vitamins related to the family of tocopherols. Tocopherol and tocotrienol are well recognized for their antioxidative effect (Kamal 1996). This effect depends primarily on the phenolic group in the chromanol ring, rather than the side chain (Burton \& Ingold 1989). The trolox equivalent antioxidant capacity (TEAC) assay is widely applied to assess the amount of radicals that can be scavenged by an antioxidant, i.e. the antioxidant capacity (Lien et al. 1999).

The present investigation was focused on the preparation of palm esters-based emulsions of tocotrienol and to characterise the rheological properties of the emulsion systems. Furthermore, assessment of the in vitro antioxidant activity of esters-based palm was done by the TEAC assay.

\section{EXPERIMENTAL}

\section{Materials}

POEs was prepared in the laboratory according to the method of Keng et al. (2009) Sorbitan monooleate $\left(\operatorname{Span}^{\circledR} 80\right)$ and polyoxethylene (20) sorbitan monooleate (Tween ${ }^{\circledR} 80$ ) were purchased from Merck, Germany. The HLB values of sorbitan monooleate $\left(\operatorname{Span}^{\circledR} 80\right)$ and polyoxethylene (20) sorbitan monooleate (Tween ${ }^{\circledR} 80$ ) are 4.3 and 15.0, respectively. Tocotrienol (Gold Tri. E 70) was from Golden Hope Bioganic, Malaysia. Xanthan gum from Xanthomonas campestris was obtained from Fluka Chemie GmbH, France. Freshly deionized water was obtained from water deionizer, Mili-Q (Milipore, USA).

\section{Methods}

Preparation of emulsions containing tocotrienol. Emulsions were formulated using POEs containing tocotrienol as dispersed oil phase and Mili-Q water as the continuous aqueous phase. Xanthan gum was dispersed in deionized 
water at $0.8 \%(\mathrm{w} / \mathrm{w})$. Preparation of dispersed oil phase was performed by homogenizing $5 \%(\mathrm{w} / \mathrm{w})$ of surfactants into oil phase with a Polytron homogenizer (Kinematica $\mathrm{GmbH}$, Germany) rotor stator. The ratio of $\operatorname{Span}^{\circledR}$ 80 :Tween ${ }^{\circledR} 80$ was $1: 4$. The preparation was continued by adding the oil phase dropwise to the aqueous solution with continuous homogenized at 6000 r.p.m. for $5 \mathrm{~min}$. The temperature was lowered to $40^{\circ} \mathrm{C}$. At $40^{\circ} \mathrm{C}$, the active ingredient was added. The emulsions were further homogenized using ultrasonic cavitation for $5 \mathrm{~min}$. The sonifier tip horn was adjusted to $2 \mathrm{~cm}$ below the surface of a $100 \mathrm{ml}$ sample.

Rheology measurement. A stress/rate controlled Kinexus Rheometer (Malvern Instrument, UK) with a temperature controller, was used to measure the rheological properties of the emulsion. The measurements were performed at $25.0 \pm 0.1^{\circ} \mathrm{C}$ with $4^{\circ} / 40 \mathrm{~mm}$ cone and plate geometry. The samples were allowed to relax for $10 \mathrm{~min}$ after being loaded to the plate before the measurement was started.

In vitro antioxidant activity. The antioxidant activity was assessed as described below. Experiments were performed on the Varian Cary 50 UV-Vis spectrophotometer (Varian, Australia). Trolox (2.5, 5.0, 10.0, $15.0 \mu \mathrm{M})$ was prepared in ethanol. Ascorbic acid was prepared in $18 \mathrm{M} \Omega$ water to a concentration of 10.0, 15.0, 20.0 $\mu \mathrm{M}$ and $\alpha$-tocopherol in ethanol at 10.0, 15.0, 20.0 $\mu \mathrm{M}$. ABTS, 2,2'-azinobis (3-ethylbenzothiazoline-6-sulphonic acid) diammonium salt was dissolved in water to a $7 \mathrm{mM}$ concentration. ABTS radical cation $\left(\mathrm{ABTS}^{\circ+}\right.$ ) was produced by reacting ABTS stock solution with $2.45 \mathrm{mM}$ potassium persulphate (final concentration) and allowing the mixture to stand in the dark at room temperature for 12 $\mathrm{h}-16 \mathrm{~h}$ before use. The radical was stable in this form for more than two days when stored in the dark at room temperature. ABTS $^{+}$solution was then diluted with ethanol to an absorbance of $0.70( \pm 0.02)$ at $734 \mathrm{~nm}$ and equilibrated at $30^{\circ} \mathrm{C}$ (Roberta et al. 1999).

Diluted ABTS ${ }^{+}$solution $(1.0 \mathrm{ml})\left(\mathrm{A}_{734 \mathrm{~nm}}=\right.$ $0.700 \pm 0.020)$ was added to $10 \mu \mathrm{l}$ of antioxidant compounds or Trolox standards in ethanol. The absorbance of the sample was taken at $30^{\circ} \mathrm{C}$ every min after initial mixing up to $6 \mathrm{~min}$. An appropriate solvent blanks were run in each assay. All determinations were carried out three times, and in triplicate, on each occasion and at each separate concentration of the standard and samples. The percentage inhibition of absorbance at $734 \mathrm{~nm}$ was calculated and plotted as a function of concentration of antioxidants and of Trolox for the standard reference data (Roberta et al. 1999).

\section{RESULTS AND DISCUSSION}

\section{Rheological Properties of Emulsions System}

Steady-state flow: The sensitivity of emulsions to shearing. The sensitivity of these emulsions to shearing was tested in steadystate flow. The greater the yield stress $\sigma_{Y}$ the more brittle the emulsion, and this leads to believe that the emulsion either undergoes disorganization of its structure or takes longer to recover its initial states. Figures 1 and 2 summarize the yield stress data as a function of acoustic amplitudes (\%) and surfactant concentration $[\%(\mathrm{w} / \mathrm{w})]$, respectively. The yield stress of the emulsions increased with increasing acoustic amplitudes. The increase in acoustic amplitudes (20\% to $100 \%)$ led to decrease in mean droplet size. The decrease of droplet size leads to the increase in the total droplet surface area. When the total surface area of the droplet increased, the strength of the attractive force will also increase. Thus, greater stress is required to initiate flow when high attractive force is holding the droplets resulting high viscosity with high yield stress (Pal 1996). Mean droplet size was another factor affecting the flow behaviour of the emulsion. 


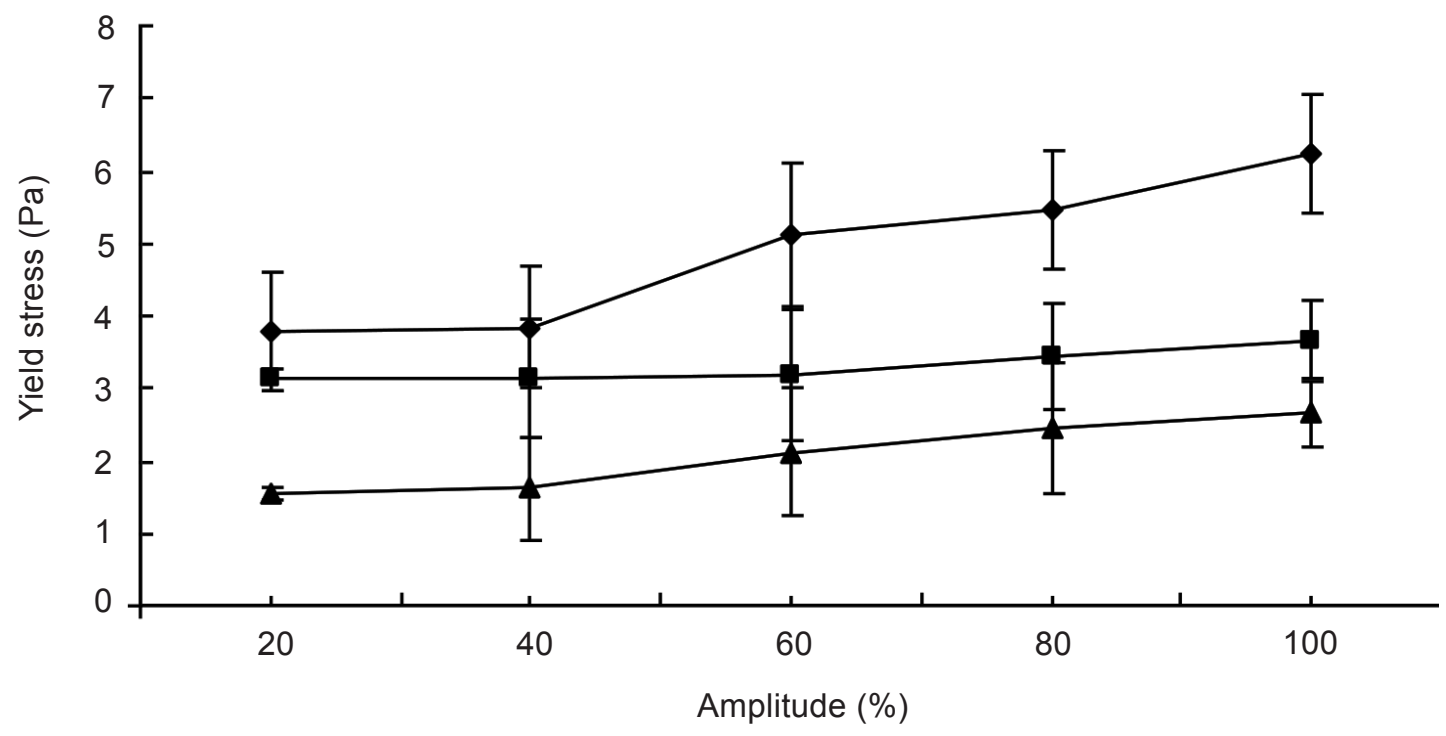

Figure 1. The yield stress of the emulsions as a function of acoustic amplitudes and oil phase

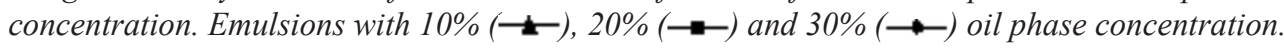

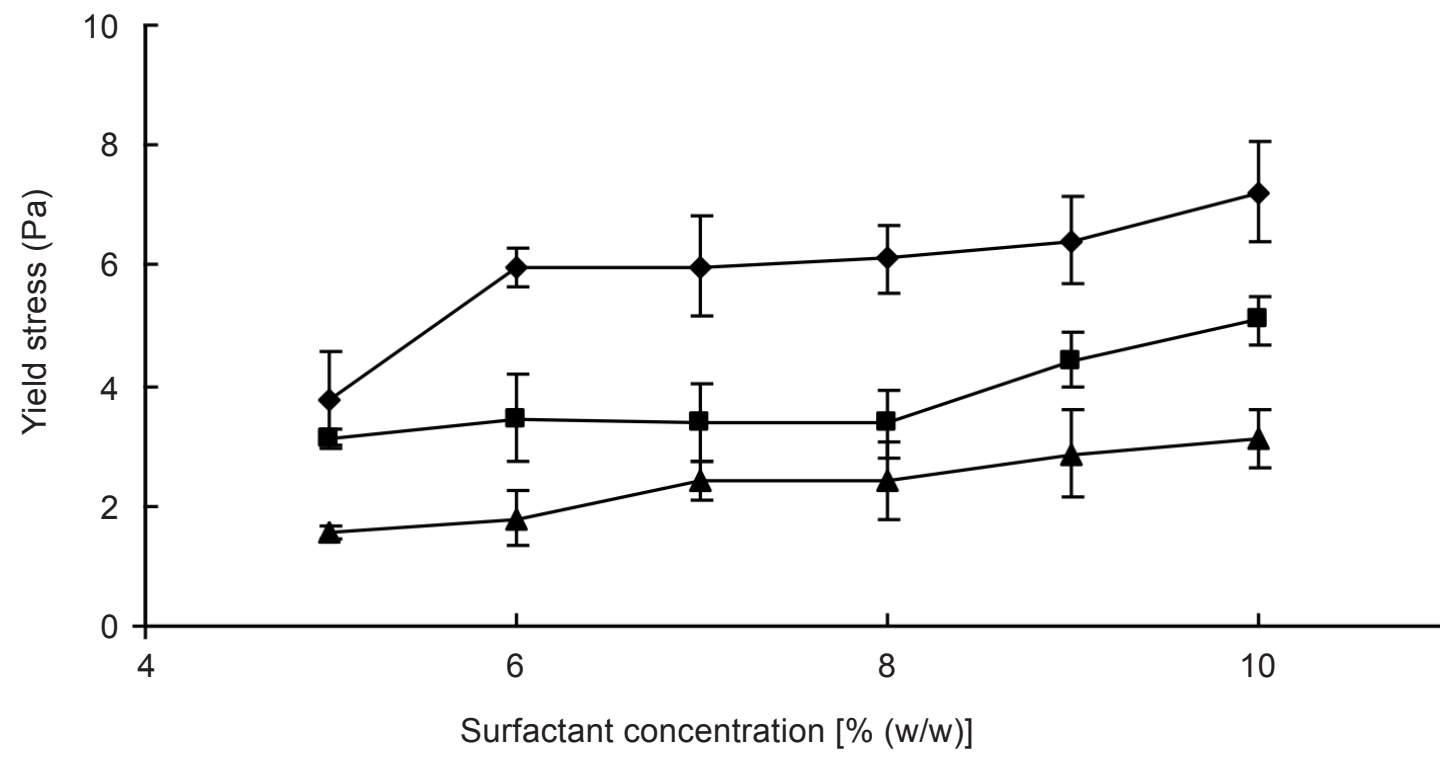

Figure 2. The yield stress of the emulsions as a function of surfactant concentration. Emulsions with $10 \%(\rightarrow-), 20 \%(\rightarrow-)$ and $30 \%(\rightarrow)$ oil phase concentration. 
The yield stress of the emulsions increased with surfactant concentration (Figure 2) indicating structural integrity arising from the strong colloidal interaction between the droplets. The yield stress is the stress that has to be overcome before the emulsion starts to flow (Barnes 1999). The system with higher surfactant concentration tends to form a denser interfacial layer, which is incompressible (Hamill \& Petersen 1966). Hence, the droplets in such sterically stabilized system are usually characterized as 'hard sphere' (McClements 1999). The strength of interaction forces (mainly the attractive and repulsive interactions) between the droplets for the hard sphere system (high surfactant concentration system) was relatively greater than the one with lower surfactant concentration. In the absence of the strong sterically repulsive effect, the droplets in the emulsions system with lower surfactant concentration were able to pack more efficiently even at low shear. Therefore, the droplets were aligning themselves easily with the shear field to initiate flow. This explained that the increase in surfactant concentration led to increase in yield stress of emulsion system.

Oil phase concentration in an emulsion system is another factor affecting the flow behaviour (Akhtar et al. 2005). The attractive force is one of the colloidal interactions which play an important role in the increase in viscosity and yield stress. The magnitude of viscosity and yield stress depend on the strength of the attractive force between the droplets (Pal 1996). Higher strength of attractive force between the droplets leads to increase in viscosity and yield stress.

\section{Shear Stress versus Shear Rate Profile}

The shear stress - shear rate profile of stabilized emulsions are depicted in Figure 3. Much like viscosity - shear rate profile, the rate of change of shear stress depends largely on the rate of change of shear rate. As depicted in Figure 3, at zero shear rate the shear stress responses are not zero. This suggested that these emulsions were shear thinning non-ideal plastic-like material, with yield stress $\left(\sigma_{Y}\right)$ response. In other words, they behaved like pseudoplastic material, which implied that flow can only be induced on these emulsions with the application of certain minimum amount of stress called yield stress. Figure 3 shows that above the yield stress these samples assume a linear shear stress - shear rate relationship. This in turn suggested that, these emulsions did not follow ideal Newtonian flow behaviour even at high shear rate domain. By contrast, the shear stress-shear rate relationship increased exponentially with a certain power law exponent at low-shear rate domain below yield stress, suggesting that the flow behaviour of these emulsions resembled that of plastic-like material at these low shearrate domain.

As far as the effect of concentration on yield stress was concerned, these profiles suggested that yield stress increased monotonically with surfactant concentration. This in turn indicated that all samples examined here exhibited non-Newtonian model type fluid behaviours, implying that the viscoelastic force dominated over the elastic force, and that the emulsions under investigation underwent structural deformation with shear rate irrespective of surfactant concentration. The increase in the yield stress as a function of surfactant concentration further indicated that emulsions with higher surfactant concentration possess higher degree of material structuring as opposed to lower surfactant concentration. This also means that emulsions with higher surfactant concentration offer a larger resistance to external force before they started flowing. This in turn suggested that emulsions stabilized with higher surfactant concentration undergo a greater degree of deformation under applied shear in comparison to emulsions stabilized with lower surfactant concentration. 


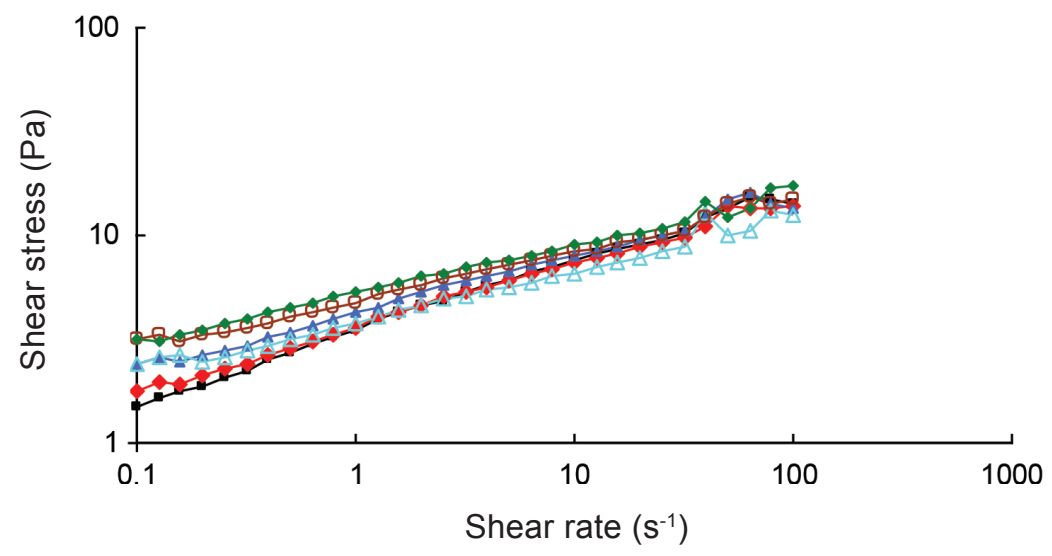

(a)

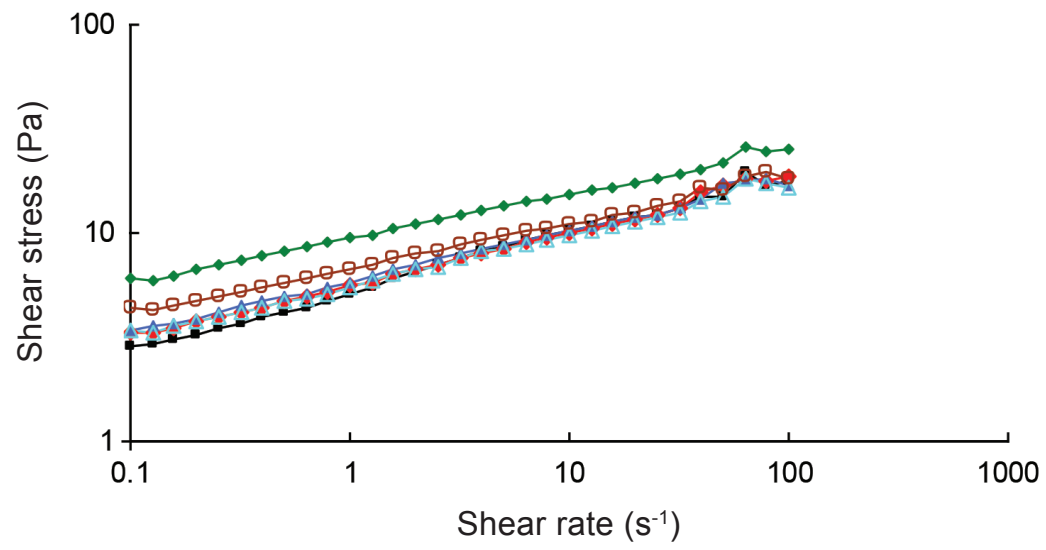

(b)

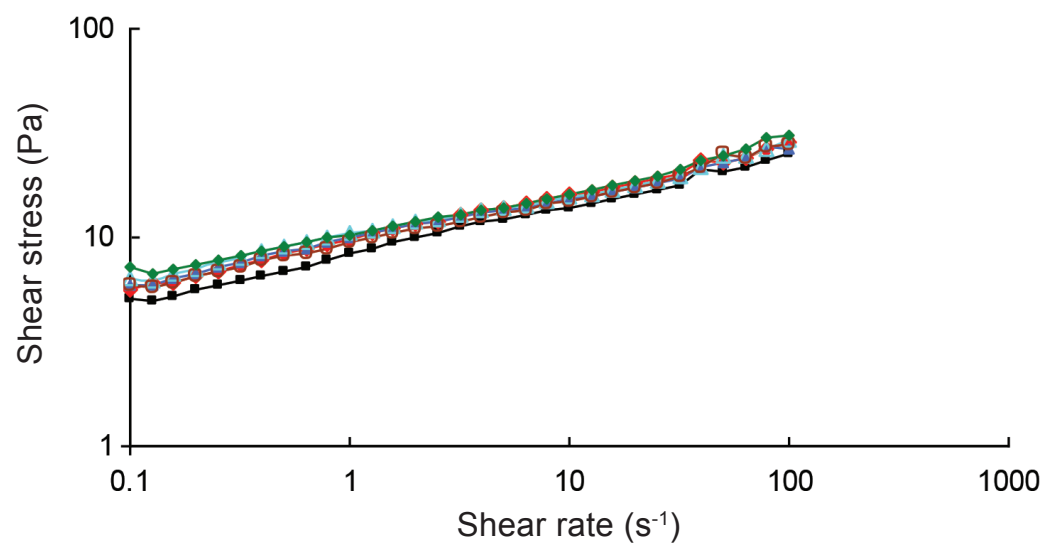

(c)

Figure 3. Effect of shear rate of emulsions on the shear stress for (a) $10 \%$ (b) $20 \%$ (c) $30 \%$ oil phase concentration. Surfactant concentration: $5 \%(\rightarrow-) ; 6 \%(-)$; $7 \%(\multimap) ; 8 \%(\multimap) ; 9 \%(\multimap)$ and $10 \%(\multimap)$. 


\section{Oscillatory Measurements: Strain Sweep Profile}

A critical strain $\left(\gamma_{c}\right)$ is the maximum applied strain where the emulsion still gives a linear response to shear stress with constant elastic modulus. The critical strain, $\gamma_{c}$ of emulsions in different oil phase concentration is shown in Figure 4. The $\gamma_{c}$ of the emulsions increased with the oil volume fraction and surfactant concentration. The $\gamma_{c}$ was increased $50 \%$, $46 \%$ and $86 \%$ as the surfactant concentration was increased from $5 \%$ to $10 \%(\mathrm{w} / \mathrm{w})$ for emulsions with $30 \%, 20 \%$ and $10 \%(\mathrm{w} / \mathrm{w})$ oil phase concentration, respectively. On the other hand, the $\gamma_{c}$ increased more than $100 \%$ when the oil concentration was increased from $10 \%$ to $30 \%(\mathrm{w} / \mathrm{w})$.

The increase of critical strain of emulsion with $30 \%(\mathrm{w} / \mathrm{w})$ oil phase concentration when the surfactant concentration was increased implied that the highly packed droplets have developed a strong structure due to the high interdroplet interaction between the droplets which corresponded to the droplet size and droplet concentration of the emulsions system. Since the strength of the interdroplet interactions corresponded to the mean separation distance between the droplets, the highly packed emulsion system will therefore has greater interdroplet interaction forces. The high interdroplet interaction strength was able to hold the droplets and withstand the large deformation forces applied during the strain sweep test.

The strain sweep profiles also provided information about the elastic component of the emulsions. Figures 5 and 6 show increasing trends in the elastic modulus $\left(G^{\prime}\right)$ of the emulsions with surfactant and oil concentration indicating that the interactions between droplets are relatively strong. A trend of increasing elastic modulus accompanying the increased of $\gamma_{c}$ was observed. The cohesive energy $\left(E_{c}\right)$ within the linear viscoelastic regime for when $G^{\prime}$ is in phase with the applied strain amplitude can also be obtained (Bossard et al. 2007), as shown below:

$$
E_{c}=\int_{0}^{\gamma c} \sigma d \gamma
$$

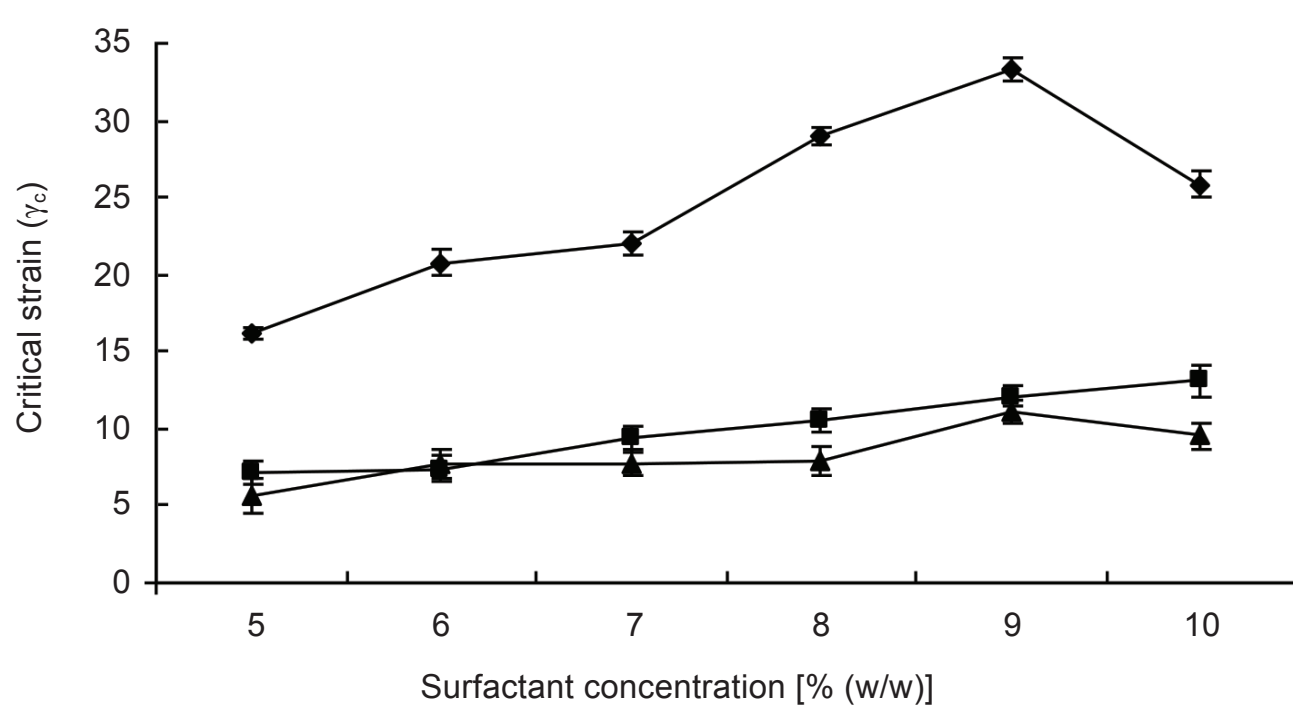

Figure 4. The critical strain, $\gamma_{c}$ of the emulsions as a function of surfactant concentration. Emulsions

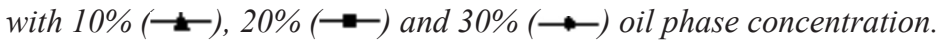




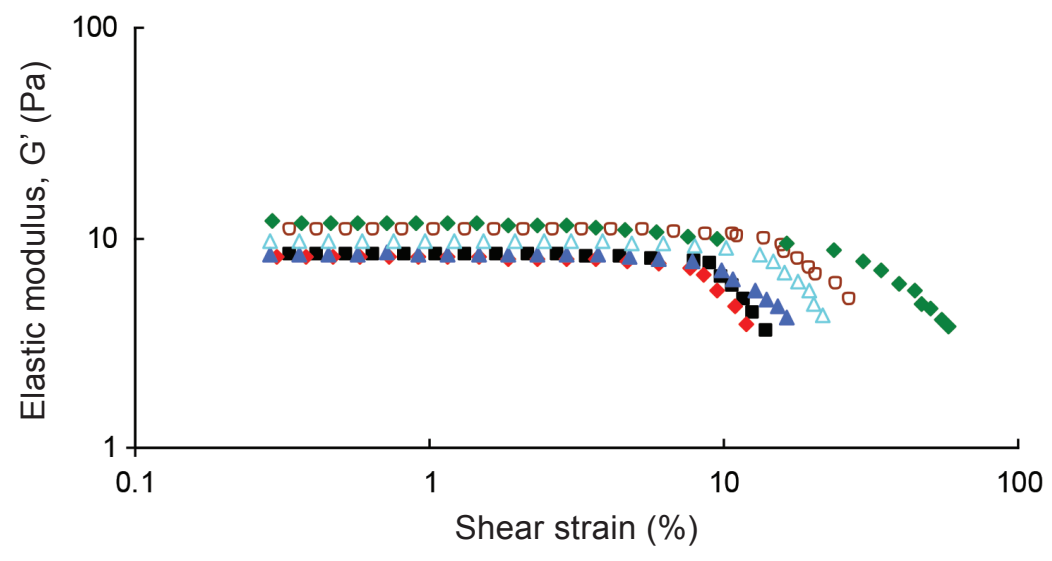

(a)

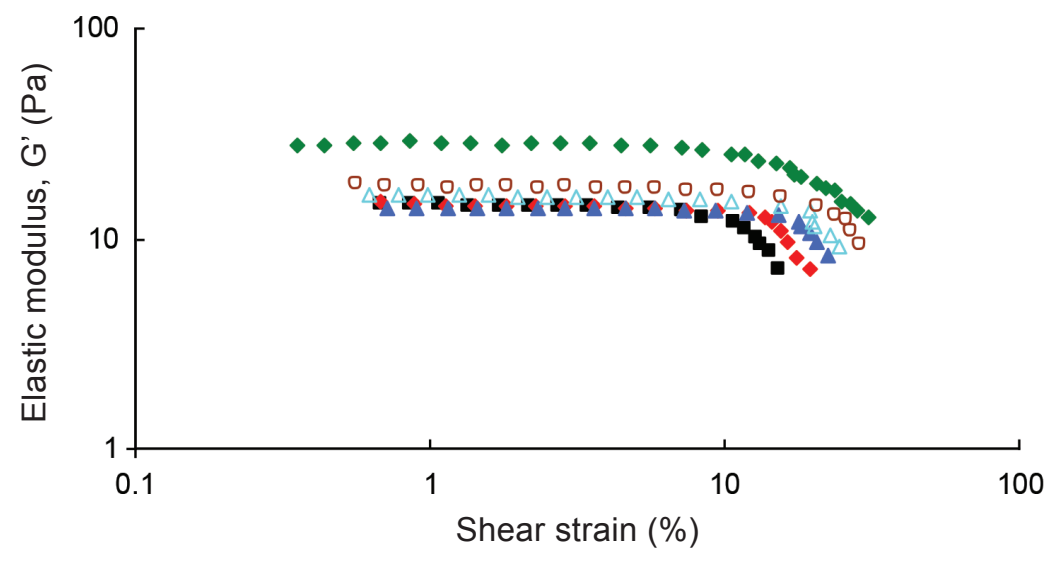

(b)

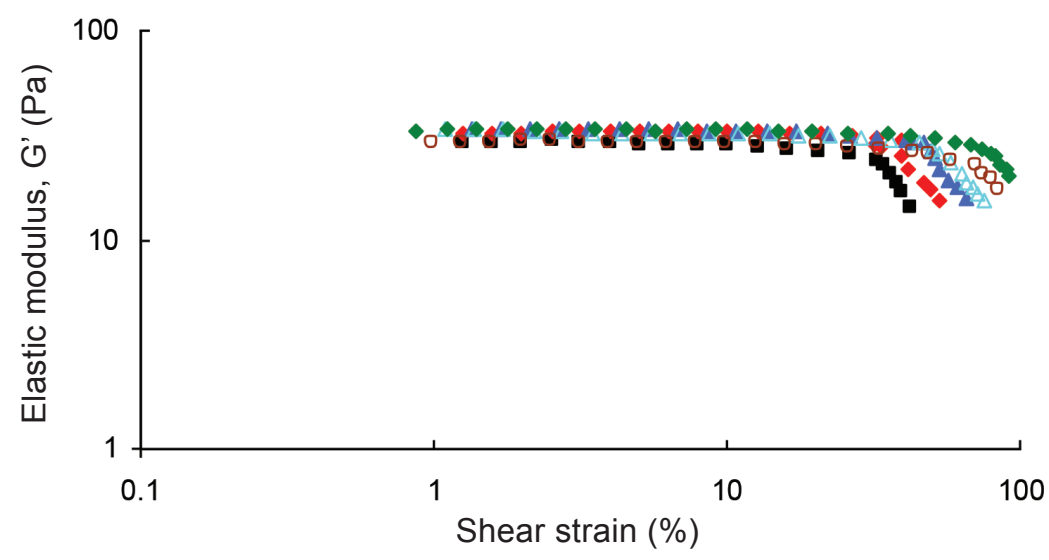

(c)

Figure 5. The linear viscoelastic region of the emulsions with a series of surfactant concentration

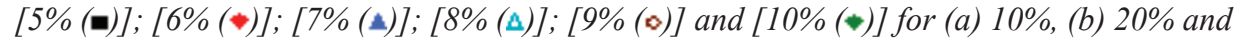
(c) $30 \%$ oil phase concentration. 
When $\sigma$ equals $G^{\prime}$ in the linear viscoelastic region, the cohesive energy is defined as:

$$
E_{c}=\frac{1}{2} \gamma^{2}{ }_{c} G^{\prime}
$$

Tadros (2004) explained that the cohesive energy was related to the structure of the emulsion system, which correlated to the droplet size and number of contact area between the droplets. The droplet concentration and the packing of the droplets influenced the strength of the cohesive force. As discussed before, the number of droplets was increased as the oil concentration was increased from $10 \%$ to $30 \%(\mathrm{w} / \mathrm{w})$ at fix surfactant concentration. As a result, the number of contacts area within the droplets increased. Thus, increases in the cohesive energy of the emulsions system were observed.

$E_{c}$ ranged from a low $0.13 \mathrm{~J} / \mathrm{m}^{3}$ for emulsion with $5 \%(\mathrm{w} / \mathrm{w})$ surfactant concentration in $10 \%(\mathrm{w} / \mathrm{w})$ oil phase concentration to a high of $15.88 \mathrm{~J} / \mathrm{m}^{3}$ for emulsion with $10 \%(\mathrm{w} / \mathrm{w})$ surfactant concentration in $30 \%(\mathrm{w} / \mathrm{w})$ oil phase concentration. $E_{c}$ was low for emulsion with $5 \%(\mathrm{w} / \mathrm{w})$ surfactant concentration in $10 \%(\mathrm{w} / \mathrm{w})$ oil phase concentration as the elasticity was low (Figure 6 ). The higher the cohesive energy, the more stable a system was as the elastic strength was basically a measure of the strength of the internal structure. This in turn demonstrated that the emulsion samples under examination were stable systems, and that the stability of these emulsions systems was enhancing with decreasing droplet size.

Figure 7 show that the cohesive energy increased significantly with surfactant concentration especially for the emulsions with $30 \%(\mathrm{w} / \mathrm{w})$ oil phase concentration. The dramatic increase of cohesive force was due to the highly packed systems related to the interdroplet interactions that had been previously discussed.

\section{In vitro Antioxidant Activity}

The concentration-response curve for six sequentially and separately prepared stock standards of Trolox was illustrated in

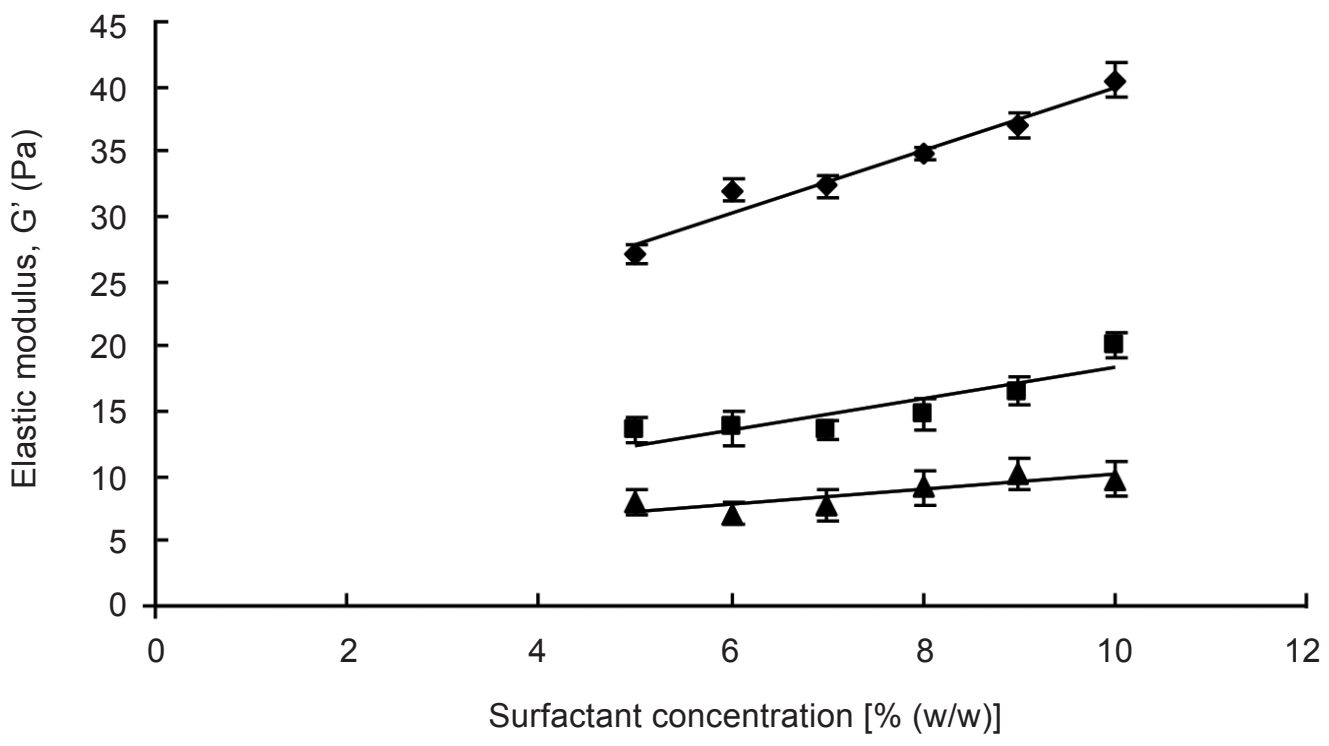

Figure 6. The elastic modulus of the emulsions as a function of surfactant concentration. Emulsions with $10 \%(\rightarrow), 20 \%(\rightarrow-)$ and $30 \%(\rightarrow)$ oil phase concentration. 


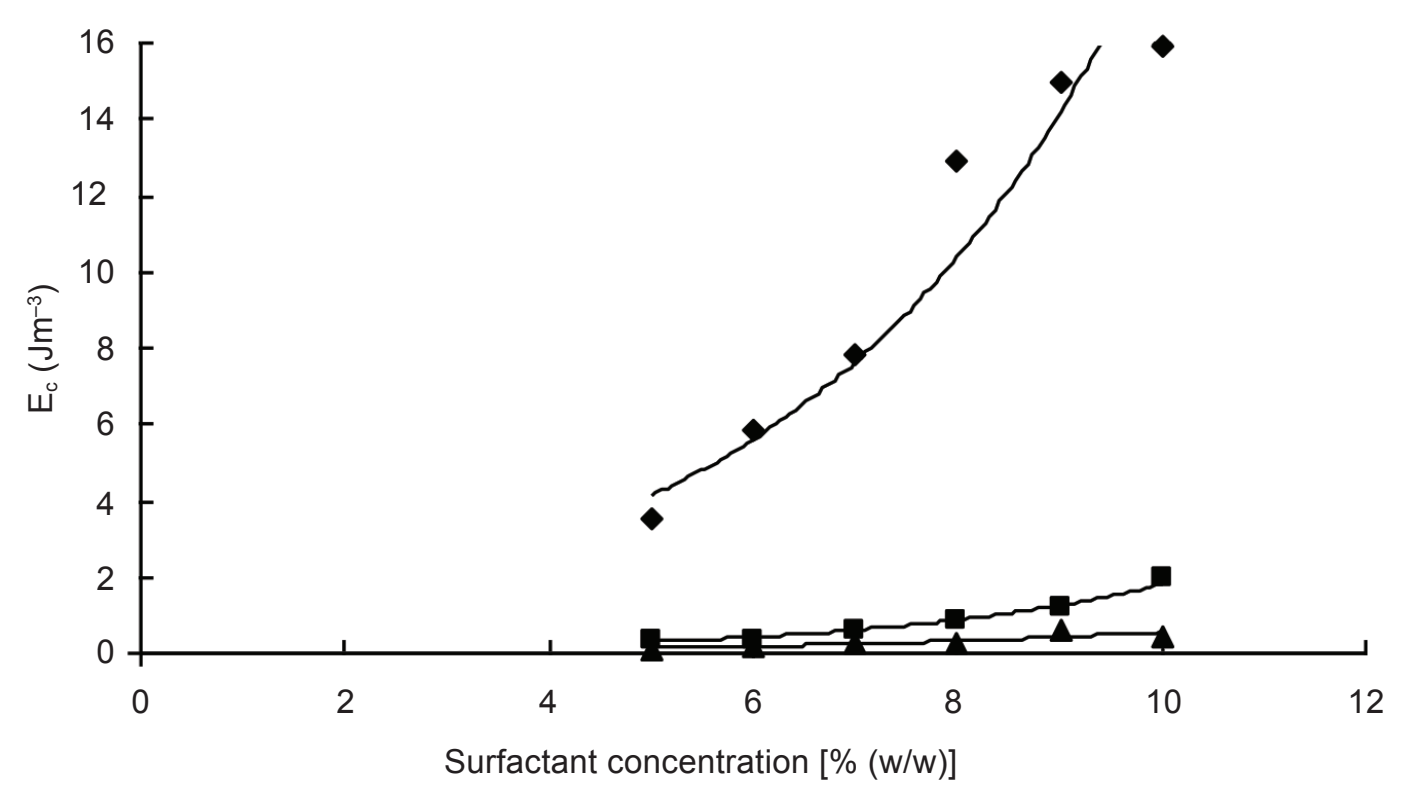

Figure 7. The cohesive energy of the emulsions as a function of surfactant concentration and oil concentration. Emulsions with 10\% (-), 20\% (-) and 30\% $\rightarrow$ ) oil phase concentration.

Figure 8. Trolox, the water-soluble analogue of $\alpha$-tocopherol was known for its high radical scavenging activity and was therefore frequently used as a model compound. It was commonly used as a reference antioxidant, in which the radical scavenging activity was expressed as Trolox equivalents. The higher the concentration of Trolox used, the more the absorbance of ABTS radicals was suppressed. The selected absorption wavelength was at 734 $\mathrm{nm}$ because $\mathrm{ABTS}^{\bullet+}$ has maximum absorption at $734 \mathrm{~nm}$ and most antioxidant does not absorb light at $734 \mathrm{~nm}$. Figure 9 illustrates the effects of the duration of interaction of specific antioxidants on the suppression of the absorbance of the $\mathrm{ABTS}^{-+}$radical cation at $734 \mathrm{~nm}$ for Trolox, the standard reference compound, compared with $\alpha$-tocopherol, ascorbic acid and emulsion samples containing tocotrienol.

The results demonstrated that the reaction with $\mathrm{ABTS}^{\bullet+}$ was completed after $1 \mathrm{~min} .30 \%$ dispersed phase emulsion containing tocotrienol $(15 \mu \mathrm{M})$ has minimum absorption than in $20 \%$ and $10 \%$ dispersed phase emulsion containing tocotrienol $(15 \mu \mathrm{M})$ with ABTS $^{\cdot+}$ within 6 min. The absorption at wavelength $734 \mathrm{~nm}$ for $\mathrm{ABTS}^{\bullet+}$ radical cation was 0.73 followed by $\mathrm{ABTS}^{\bullet+}$ radical cation in ascorbic acid and tocopherol which gave 0.42 and 0.28 , respectively. From the results, 30\% dispersed phase emulsion containing tocotrienol $(15 \mu \mathrm{M})$ revealed better antioxidant properties by presenting lower absorbance value as compared the standard reference compound, Trolox. The extent of inhibition of the absorbance of the $\mathrm{ABTS}^{\circ+}$ was plotted as a function of concentration in order to determine the TEAC that can be assessed as a function of time. The dose-response curve obtained by analysis of a range of concentrations of antioxidant compounds and Trolox standards was plotted as the percentage inhibition of the absorbance of the $\mathrm{ABTS}^{\cdot+}$ solution as a function of concentration of antioxidant (Figure 10). The result showed that $30 \%$ dispersed phase emulsion containing tocotrienol $(15 \mu \mathrm{M})$ has an inhibitory effect, a maximum concentration of $20 \mu \mathrm{M}$ reducing $\mathrm{ABTS}^{\bullet+}$ by about $93 \%$. 


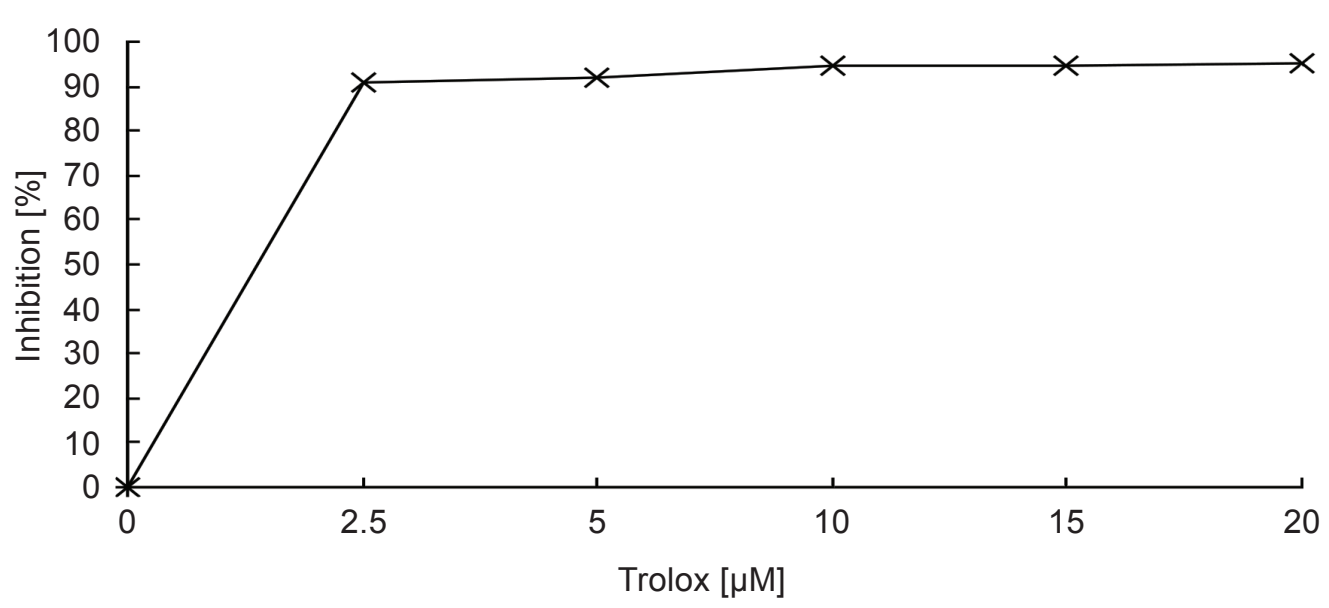

Figure 8. Concentration-response curve for the absorbance at $734 \mathrm{~nm}$ for $\mathrm{ABTS}^{++}$as a function of concentration of standard Trolox solution.

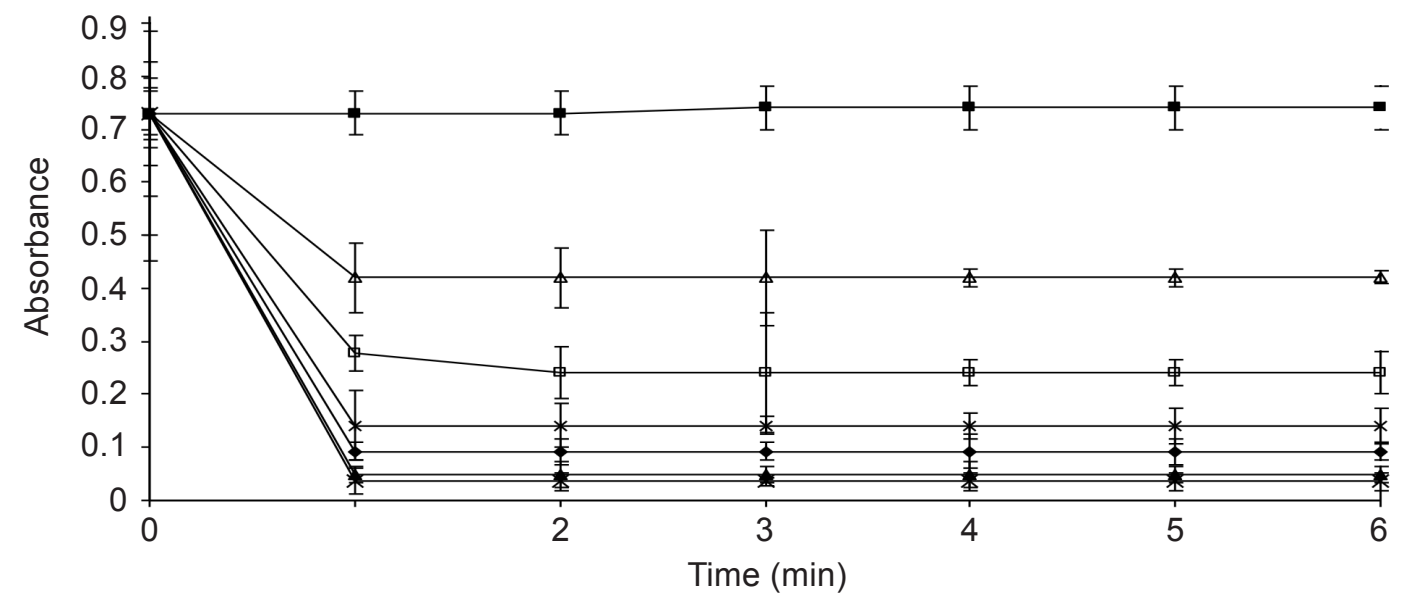

Figure 9. Effects of time on the suppression of the absorbance of the ABTS ${ }^{++}$; $A B T S^{++}(--)$;

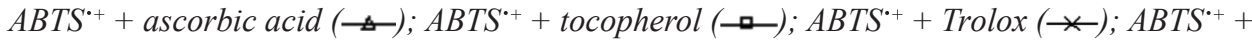
$10 \%$ disperse phase containing tocotrienol ( - ); $A B T S^{++}+20 \%$ disperse phase containing tocotrienol $(\rightarrow)$ and $A B T S^{++}+30 \%$ disperse phase containing tocotrienol $(\rightarrow)$.

The concentration of the samples used in the determination is $15 \mu \mathrm{M}$.

From the result, $20 \%$ dispersed phase emulsion containing tocotrienol $(15 \mu \mathrm{M})$ appears to exert a slightly greater inhibitory effect on the $\mathrm{ABTS}^{\cdot+}$ than on that of $10 \%$ dispersed phase emulsion. The presence of the $30 \%$ dispersed phase emulsion containing tocotrienol $(15 \mu \mathrm{M})$ enhanced its inhibitory effect on the $\mathrm{ABTS}^{\circ+}$ since $20 \mu \mathrm{M}$ of $20 \%$ dispersed phase emulsion containing tocotrienol $(15 \mu \mathrm{M})$ and $10 \%$ disperse phase emulsion containing tocotrienol $(15 \mu \mathrm{M})$ only produced $87.5 \%$ and $82.8 \%$ inhibition; respectively of the ABTS assay system. The antioxidant activity could be expressed in terms of the 
total contribution to the antioxidant activity over the time range studied by calculating the TEAC, the gradient of the plot of the percentage inhibition of absorbance vs. concentration plot for the antioxidant in question is divided by the gradient of the plot for Trolox. This gives the
TEAC at the specific time points, $1 \mathrm{~min}, 4 \mathrm{~min}$ and 6 min (Figure 11).

From the results, it was found that all samples tested were able to scavenge $\mathrm{ABTS}^{\bullet+}$ radical cation. Their antioxidant activities

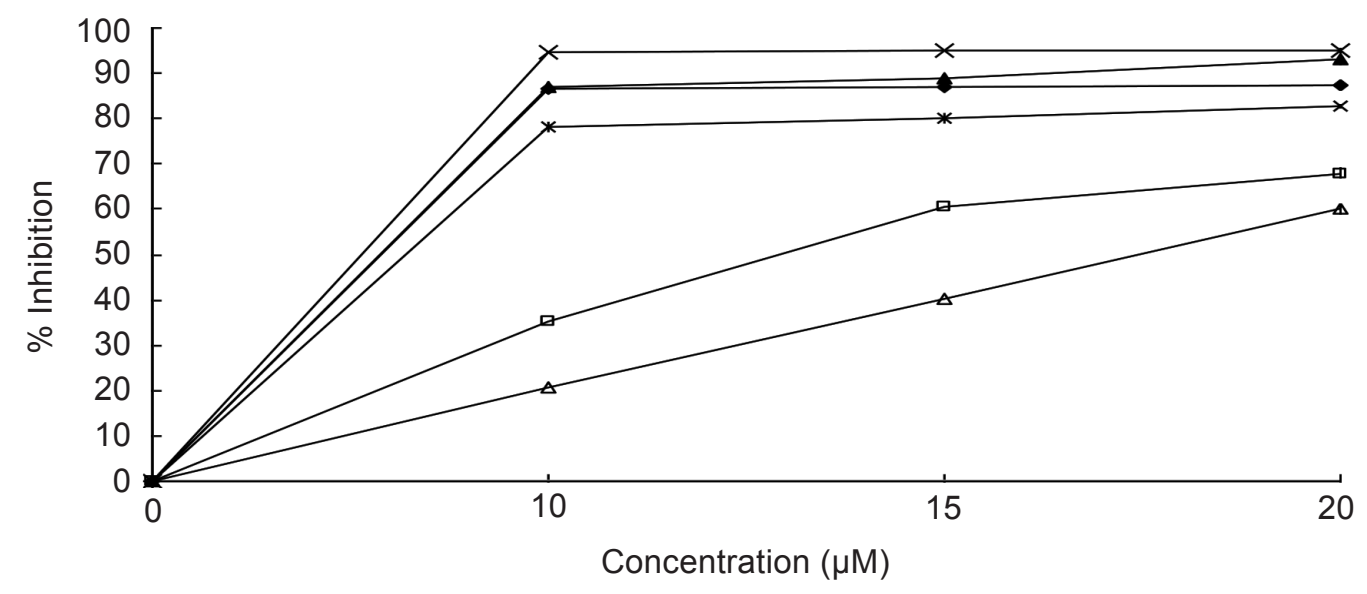

Figure 10. Effects of concentration of the antioxidant on the inhibition of the $A B^{\bullet^{+}}$; $A B T S^{\cdot+}$

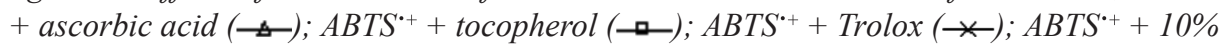
dispersed phase containing tocotrienol (-*); ABTS ${ }^{++}+20 \%$ dispersed phase containing tocotrienol $(\rightarrow)$ and ABTS ${ }^{\cdot+}+30 \%$ dispersed phase containing tocotrienol $(\rightarrow)$. The concentration of the samples used in the determination is $15 \mu \mathrm{M}$.

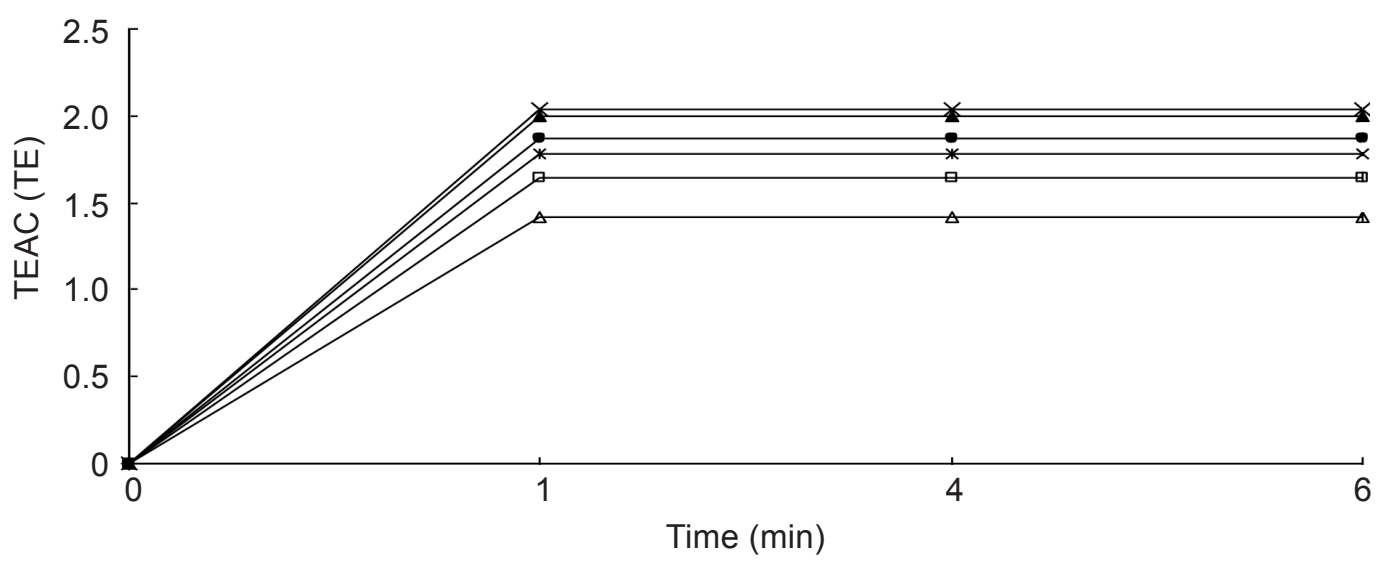

Figure 11. Profile of the variation of gradient of the percent inhibition vs. concentration plot of each antioxidant at 1, 4 and 6 min: ABTS $^{\cdot+}+$ ascorbic acid (ـ); ABTS $^{\cdot+}+$ tocopherol (- -$) ;$ ABTS $^{\cdot+}+$ Trolox (־); ABTS $^{++}+10 \%$ dispersed phase containing tocotrienol (- $\left.\longleftarrow\right) ; A B T S^{++}+20 \%$ dispersed phase containing tocotrienol $(\rightarrow)$ and $\mathrm{ABTS}^{\cdot+}+30 \%$ dispersed phase containing tocotrienol $(\rightarrow)$.

The concentration of the samples used in the determination is $15 \mu \mathrm{M}$. 
measured at 6 min and expressed as the TEAC value or $\mathrm{IC}_{50}$ were relatively low as compared to Trolox. The higher TEAC values implied higher antioxidant capability. The TEAC at $6 \mathrm{~min}$ of $30 \%$ dispersed phase emulsion containing tocotrienol $(15 \mu \mathrm{M})$ was 2.00 whereas the TEAC values of tocopherol and ascorbic acid were 1.64 and 1.42 respectively. Tocotrienol in $30 \%$ dispersed phase appeared to have a much higher TEAC value than tocopherol and ascorbic acid in $15 \mu \mathrm{M}$ concentrations, respectively. This means that tocotrienol can be qualified as the best antioxidant compared to tocopherol and ascorbic acid with the TEAC assay.

\section{CONCLUSIONS}

The results obtained in the present work showed that emulsions containing palm esters-based as oil phase was a suitable carrier system for the incorporation of tocotrienol with reasonably high values of yield stress, suggesting the stabilization of emulsions. In vitro antioxidant activity using TEAC assay was applied to assess the total amount of radicals that could be scavenged by antioxidant samples. The findings indicated that tocotrienol in emulsion with $30 \%(\mathrm{w} / \mathrm{w})$ dispersed phase exhibited greater inhibitory effects on the ABTS ${ }^{+}$. Results were encouraging and it substantiated the role of palm esters-based emulsions containing tocotrienol as an effective antioxidant therapy.

\section{ACKNOWLEDGMENTS}

We acknowledge the financial support from University Putra Malaysia.

\section{Date of submission: June 2014 \\ Date of acceptance: October 2014}

\section{REFERENCES}

Akhtar, M, Stenzel, J, Murray, BS \& Dickinson, E 2005, 'Factors affecting the perception of creaminess of oil-in-water emulsions', Food Hydrocolloids, vol. 19, pp. 521-526.
Barnes, HA 1999, 'The yield stress - everything flows?,' Journal of Non-Newtonian Fluid Mechanics, vol. 81, pp. 133-178.

Basri, MR, Salleh, M, Ariff, AB, Mohammad, BA, Abdul Rahman, RMB \& Rahman, RNZ 2006, 'Optimization study of large-scale enzymatic synthesis of oleyl oleate, a liquid fat esters by response surface methodology', Journal of Chemistry Technology Biotechnology, vol. 81, pp. 374-380.

Bossard, F, Moan, M \& Aubry, T 2007, 'Linear and non-linear viscoelastic behaviour of very concentrated plate-like kaolin suspensions', Journal of Rheology, vol. 51, no. 6, pp. 12531270.

Bummer, R \& Godersky, S 1999, 'Rheological studies to objectify sensations occurring when cosmetic emulsions are applied to the skin', Colloids and Surfaces A: Physicochemical and Engineering Aspects, vol. 152, pp. 89-94.

Burton, GW \& Ingold, KU 1989, 'Vitamin E as in vitro and in vivo antioxidant', Ann. New York Academy Science, vol. 570, pp. 7-22.

Choi, CM \& Berson, DS 2006, 'Cosmeceuticals', in Seminars in Cutaneous Medicine and Surgery, vol. 25 , no. 3 , pp. 163-168.

Hamill, HD \& Petersen, RV 1966, 'Effects of aging and surfactant concentration on the rheology and droplet size distribution of a nonaqueous emulsion', Journal of Pharmaceutical Sciences, vol. 55, pp. 1268-1277.

Kamal, EA 1996, 'The chemistry and antioxidant properties of tocopherols and tocotrienols', Lipids, vol. 31, pp. 671-701.

Karg, RF, Boozer, CE \& Benefield, RE 1985, 'Injection molding of elastomers', Rubber World, vol. 192, pp. 14-19.

Keng, PS, Basri, M, Zakaria, MRS, Abdul Rahman, MB, Ariff, AB, Abdul Rahman, RNZ \& Salleh, AB 2009, 'Newly synthesized palm esters for cosmetics industry', Industrial Crops and Products, vol. 29, pp. 37-44.

Kikic, I, Lapasin, R, Torriano, G \& Papo, A 1979, 'Processing intermediates for high-build paints', Journal of Coatings Technology, vol. 51, pp. 29-33.

Lien, EJ, Ren, S, Bui, HH \& Wang, R 1999 , 'Quantitative structure activity relationship analysis of phenolic antioxidants', Free Radical Biology and Medicine, vol. 26, pp. 285-294. 
Lorenzo, G, Zaritzky, N \& Califano, A 2008, 'Modelling rheological properties of low-in-fat $\mathrm{o} / \mathrm{w}$ emulsions stabilized with xanthan/guar mixture', Food Research International, vol. 41, pp. 487-494.

Martin-Alfonso, MJ, Martinez-Boza, F, Partal, P \& Gallegos, C 2006, 'Influence of pressure and temperature on the flow behaviour of heavy fuel oil', Rheologcal Acta, vol. 45, pp. 357-365.

McClements, DJ 1999, Emulsion rheology in food emulsions: principles, practice and techniques, CRC Press, Boca Raton, FL.

Pal, R 1996, 'Rheology of emulsions containing polymeric liquids', in Encyclopedia of Emulsion Technology, Marcel Dekker, New York.

Roberta, R, Nicoletta, P, Anna, P, Ananth, P, Min, Y \& Catherine, RE 1999, 'Antioxidant activity applying an improved ABTS radical cation decolourization assay', Free Radical Biology and Medicine, vol. 26, pp. 1231-1237.
Solans, C, Esquena, J, Forgiarini, AM, Morales, D, Izquierdo, P, Azemar, $\mathrm{N} \&$ Garcia-Celma, MJ 2003, 'Nanoemulsions: formation, properties and applications', Surfactant Science Series, vol. 109, pp. 525-554.

Tadros, T, Izquierdo, P, Esquena, J \& Solans, C 2004, 'Formation and stability of nano-emulsions', Journal of Colloid and Interface Science, vol. 108-109, pp. 303-318.

Tanaka, R, Hirose, S \& Hatakeyama, H 2008, 'Preparation and characterization of polyurethane foams using a palm oil-based polyol', Bioresource Technology, vol. 99, pp. 3810-3816.

Wu, H, Ramachandran, C, Weiner, N \& Roessler, B 2001, 'Topical transport of hydrophilic compounds using water-in-oil nanoemulsions', International Journal of Pharmaceutics, vol. 22, pp. 63-75.

Zumalacarregui, L, Vazquez, M, Estevez, T, Aguilera, A \& Hardy, E 2004, 'Rheological studies of interferon creams', Applied Rheology, vol. 14, pp. 251-255. 